\title{
Time Dimension and Ordinary Cosmic Energy Density Are Fractal Effects
}

\author{
Mohamed S. El Naschie \\ Dept. of Physics, Faculty of Science, University of Alexandria, Alexandria, Egypt \\ Email: Chaossf@aol.com
}

How to cite this paper: El Naschie, M.S. (2018) Time Dimension and Ordinary Cosmic Energy Density Are Fractal Effects. Journal of Quantum Information Science, 8, 47-51.

https://doi.org/10.4236/jqis.2018.82004

Received: March 30, 2018

Accepted: March 31, 2018

Published: April 3, 2018

Copyright $\odot 2018$ by author and Scientific Research Publishing Inc. This work is licensed under the Creative Commons Attribution International License (CC BY 4.0).

http://creativecommons.org/licenses/by/4.0/

\begin{abstract}
In a short, neat and credible analysis, it is established that time is a fractal effect of the Cantor set-like topology of micro spacetime. This effect as well as the ordinary cosmic energy density of the universe is shown to be a direct consequence of Hardy's probability of quantum entanglement. Finally and as a general conclusion, we point out the importance of understanding the fractal origin of time as well as spacetime for resolving certain types of paradoxes arising in quantum information science.
\end{abstract}

\section{Keywords}

Random Cantor Sets, E-Infinity, Einstein Spacetime, Cantorian Spacetime, Penrose Universe, 'thooft Renormalon, Dark Energy, Ordinary Energy Density, Correlation, Hardy's Quantum Entanglement

\section{Analysis}

To show how the title of this short letter is true, we start by using stringent mathematics to demonstrate how the time dimension is actually a fractal effect that may be called a real and persistent illusion without being inconsistent or esoteric [1]-[15]. We know from many previous publications that the average topological dimension of spacetime is given by the formula [10]-[15]

$$
\langle n\rangle=\frac{1+d_{c}^{(o)}}{1-d_{c}^{(o)}}
$$

where $d_{c}^{(0)}$ is the Hausdorff dimension of the backbone fractal-Cantor unit set forming the space of the physical-mathematical E-infinity theory [12]-[19]. Setting $d_{c}^{(O)}$ equal to the rational value $d_{c}^{(O)}=3 / 5=0.6$ [10] [11] [12], one finds 


$$
\begin{aligned}
\langle n\rangle & =\frac{1+(3 / 5)}{1-(3 / 5)} \\
& =\frac{(8 / 5)}{(2 / 5)} \\
& =4
\end{aligned}
$$

The result means that for $d_{c}^{(O)}=3 / 5=0.6$ which is the first rational approximation found from continued fraction expansion of the irrational golden mean $\phi=(\sqrt{5}-1) / 2=0.618033989$ one finds the dimensionality of Einstein's spacetime [20] [21]. On the other hand, we know that the average $d_{c}^{(O)}$ is given not by $d_{c}^{(O)}=0.6$ but by $\left\langle d_{c}^{(O)}\right\rangle=1 / 2$ which is equal to $\left\langle d_{c}^{(o)}\right\rangle=\phi-k / 10=0.618033-0.018033989=1 / 2$ as well known from the E-infinity interpretation of Sir. R. Penrose twistor theory [22]. Inserting $\left\langle d_{c}^{(O)}\right\rangle=1 / 2$ in $\langle n\rangle$ one finds [3] [8] [10] [13]

$$
\langle n\rangle=\frac{1+(1 / 2)}{1-(1 / 2)}=3
$$

which is the topological dimensionality of classical Newtonian space $[3,8,13]$. Remembering that $d_{c}^{(O)}=0.6$ is the first approximation of $d_{c}^{(O)}=\phi$ and that $\phi$ is nothing but $\left(d_{c}^{(O)}=0.6\right)$ plus 'tHooft's renormalon divided by superstring dimensionality $D=10$ [11] [12] [13] [20] [21], we can see the difference between $\langle n\rangle=4$ and $\langle n\rangle=3$ is the first effect of the irrational fractality implicated in $d_{c}^{(0)}=0.6$ [3] [10]. The preceding result could be reproduced using another manoeuvre by taking $\langle n\rangle=3$ to be $n$ and find the corresponding fractal dimension of spacetime where $d_{c}^{(O)}=1 / 2$ using the well known bijection formula of E-infinity [8] [10] [11] [12]

$$
d_{c}^{(n)}=\left(d_{c}^{(o)}\right)^{n-1}
$$

Consequently for $d_{c}^{(O)}=\left(d_{c}^{(o)}\right)=1 / 2$ and $n=3$ one finds [3] [8] [10] [12]

$$
\begin{aligned}
d_{c}^{(3)} & =[1 /(1 / 2)]^{3-1} \\
& =(2)^{2} \\
& =4
\end{aligned}
$$

That means that for $n=3$ we find $d_{c}=4$ which means that $4-3=1$ is the difference between the fractal Hausdorff dimension of spacetime, i.e. $d_{c}=4$ and the corresponding topological dimension $n=3$ so that we may interpret $\mathrm{d}=4$ not as the union of $n=3$ plus a time dimension $n$ (time) $=1$ leading to $3+1=4$ spacetime dimension but rather the difference on average between $n=3$ and $d_{c}^{(3)}=4$, i.e. the effect of hidden on average fractality [3] [8] [10] [12]. To be absolutely convinced of the soundness of our fractality interpretation, we can demonstrate yet a third derivation, this time using the true average of the Hausdorff dimensionality for $d_{c}^{(O)}=1 / 2$ which is given in E-infinity theory by [10] [12]

$$
\left\langle d_{c}\right\rangle=\frac{1}{d_{c}^{(o)}\left(1-d_{c}^{(o)}\right)}
$$


and find

$$
\begin{aligned}
\left\langle d_{c}\right\rangle & =\frac{1}{(1 / 2)(1-(1 / 2))} \\
& =(1 / 4)^{-1} \\
& =4
\end{aligned}
$$

where we saw from Equation (3) that $\langle n\rangle=3$ [3] [8] [10] [12]. Thus we can say without any reservation that time is the extra dimension arising from the fact that the spacetime manifold has an average Hausdorff dimension $\left\langle d_{c}\right\rangle=4$ larger than its embedding average topological dimension $\langle n\rangle=3$ [3] [8] [10] [12].

It is highly instructive and insightful that while we do not see but simply "feel" the time dimension, there is another effect that we can "see" and feel due to a fractality effect, namely the ordinary energy density of the cosmos [21]. Without going into the detail of why the total Hausdorff dimensionality of the universe is not given by $D=4$, nor in fact by $D=4+\phi^{3}$ of the E-infinity core but by the fractal version of Kaluza-Klein spacetime theory [20] [21] $D=\left(4+\phi^{3}\right)+1=5+\phi^{3}$, we can easily demonstrate that the $\phi^{3}$ is the Hausdorff mass of the cosmos while the 5 is the topological mass of the universe [23] [24] so that we can easily reason that the ordinary energy density of the cosmos is given by [23] [24]

$$
\phi^{3} /\left(5+\phi^{3}\right)=\phi^{5} / 2
$$

in full agreement with all actual cosmic measurements and observations [23] [24] [25]. We note on passing that $\phi^{5}$ is Hardy's probability of quantum entanglement for two quantum particles. This makes a great deal of sense because entanglement is a very strong form of correlation so that we can measure this correlated energy density with relative ease. By contrast the dark energy sector is anti-correlated, i.e. $1-\left(\phi^{5} / 2\right)$ and therefore it could not be measured in a direct way leading to the wrong supposition that dark energy does not exist [20]-[25].

From the preceding analysis and conclusion, we can draw the profound conclusion that one of the most important components of our universe and existence is its chaotic fractal dynamics, geometry and topology [8]-[16] [20]-[25]. In fact, this conclusion is the deep rationale behind our Casimir-dark energy nano reactor proposal [15] [26] [27] as well as our explanation and endorsement of NASA's electromagnetic drive which opened the possibility of fuelless interstellar spacecraft travel [28] [29]. Last but no means least, we should point out the central and important role played by understanding vital concepts or spacetime and its fractal origin for avoiding or resolving certain class of paradoxes in quantum information science such as the Hardy paradox which we have addressed in a recent paper [30].

\section{References}

[1] Majid, S. (Editor) (2008) On Space and Time. With Contributions by A. Connes, M. 
Heller, S. Majid, R. Penrose, J. Polkinghorner and Andrew Taylor. Cambridge University Press, Cambridge, UK.

[2] Laudal, O.A. (2011) Geometry of Time-Spaces. World Scientific, Singapore.

[3] El Naschie, M.S. and Progogine, I. (1996) Time Symmetry Breaking in Classical and Quantum Mechanics. Chaos, Solitons \& Fractals, (Special Issue Forward by M.S. El Naschie), 7, 441-609.

[4] Vrobel, S. (2011) Fractal Time. World Scientific, Singapore.

[5] HRH (2010) The Prince of Wales, Tony Juniper and Ian Skelly: Harmony: A New Way of Looking at Our World. Harper Collins Publications, London, UK. (See in particular page 113.)

[6] Hemenway, P. (2008) The Secret Code. Evergreen, Koln, Germany.

[7] Olsen, S. (2006) The Golden Section. Wooden Books, Glastonbury, Somerset, UK.

[8] El Naschie, M.S. (1996) Time Symmetry Breaking, Duality and Cantorian Spacetime. Chaos, Solitons \& Fractals, 7, 499-518. https://doi.org/10.1016/0960-0779(96)00007-0

[9] Stakhov, A. (2009) The Mathematics of Harmony. World Scientific, Singapore.

[10] El Naschie, M.S. (2004) A Review of E-Infinity Theory and the Mass Spectrum of High Energy Particle Physics. Chaos, Solitons \& Fractals, 19, 209-236. https://doi.org/10.1016/S0960-0779(03)00278-9

[11] El Naschie, M.S. (1998) Superstrings, Knots and Noncommutative Geometry in E-Infinity Space. International Journal of Theoretical Physics, 37, 2935-2951. https://doi.org/10.1023/A:1026679628582

[12] El Naschie, M.S. (2009) The Theory of Cantorian Spacetime and High Energy Particle Physics (An Informal Review). Chaos, Solitons \& Fractals, 41, 2635-2646. https://doi.org/10.1016/j.chaos.2008.09.059

[13] Al Athel, S. (1996) On the Dimension of Micro Spacetime. Chaos, Solitons \& Fractals, 7, 873-875. https://doi.org/10.1016/0960-0779(96)00022-7

[14] El Naschie, M.S. (2017) Elements of a New Set Theory Based Quantum Mechanics with Applications in High Energy Quantum Physics and Cosmology. International Journal of High Energy Physics, 4, 65-74.

[15] El Naschie, M.S. (2015) On a Non-Perturbative Quantum Relativity Theory Leading to Casimir-Dark Energy Nano Tech Reactor. Open Journal of Applied Science, 5, 313-324. https://doi.org/10.4236/ojapps.2015.57032

[16] El Naschie, M.S. (2017) The Aether as the Source of the Cosmic Dark Energy Sector. International Journal of Innovation in Science \& Mathematics, 5, 177-181.

[17] El Naschie, M.S. and He, J. (2018) Tesla's Dream from a Modern Quantum Space-Time View Point. Nonlinear Science Letters A, 9, 36-43.

[18] El Naschie, M.S. (2017) The Aether of Spacetime Physics Is the Empty Set of Pure Mathematics. Natural Science, 9, 289-292. https://doi.org/10.4236/ns.2017.99027

[19] El Naschie, M.S. (2017) Spacetime as a New Frontier Advanced Material with Applications in Physics, Engineering, Chemistry and Cosmology. Advances in Material Physics and Chemistry, 7, 347-353. https://doi.org/10.4236/ampc.2017.79027

[20] El Naschie, M.S. (2017) Einstein-Kaluza Combined Spacetime as the Optimal and Simplest Framework to Compute and Understand Dark Matter, Pure Dark Energy and Measurable Ordinary Energy. Natural Science, 9, 241-244.

https://doi.org/10.4236/ns.2017.98024

[21] El Naschie, M.S. (2017) From a Dual Einstein-Kaluza Spacetime to 'tHooft Renor- 
malon and the Reality of Accelerated Cosmic Expansion. Journal of Modern Physics, 8, 1319-1329. https://doi.org/10.4236/jmp.2017.88085

[22] El Naschie, M.S. (2001) On Twistors in Cantorian E-Infinity Space. Chaos, Solitons \& Fractals, 12, 741-746. https://doi.org/10.1016/S0960-0779(00)00193-4

[23] El Naschie, M.S. (2017) A Combined Heterotic String and Kähler Manifold Elucidation of Ordinary Energy, Dark Matter, Olbers's Paradox and Pure Dark Energy Density of the Cosmos. Journal of Modern Physics, 8, 1101-1118. https://doi.org/10.4236/jmp.2017.87071

[24] El Naschie, M.S. (2017) Kähler Dark Matter, Dark Energy, Cosmic Density and Their Coupling. Journal of Modern Physics, 7, 1953-1962. https://doi.org/10.4236/jmp.2016.714173

[25] Panek, R. (2012) The 4\% Universe. One World Publications, Oxford.

[26] El Naschie, M.S. (2015) A Casimir-Dark Energy Nano Reactor Design-Phase I. Natural Science, 7, 287-298. https://doi.org/10.4236/ns.2015.76032

[27] El Naschie, M.S. (2015) The Cantorian Monadic Plasma behind the Zero Point Vacuum Spacetime Energy. American Journal of Nano Research \& Application, 3, 66-70.

[28] El Naschie, M.S. (2018) NASA's EM Drive Thrust from the Forces of the Quantum Vacuum of Spacetime. Advances in Aerospace, Science \& Technology, 3, 1-9. https://doi.org/10.4236/aast.2018.31001

[29] El Naschie, M.S. (2018) A Theoretical Justification of NASA Electromagnetic Drive Based on Cosmic Dark Matter. International Journal of Engineering Innovation and Research, 7, 69-71.

[30] El Naschie, M.S. (2018) Resolution of Hardy's Paradox within Spacetime Physics and the Ithaca Interpretation of Quantum Mechanics. World Journal of Condensed Matter Physics, 8, 23-29. https://doi.org/10.4236/wjcmp.2018.82002 